\title{
Magnetic field directed assembly of two-dimensional fractal colloidal aggregates $\dagger$
}

\author{
Julie Byrom and Sibani Lisa Biswal* \\ The anisotropy of dipolar interactions can sometimes be a hindrance when assembling colloids, as it limits \\ the diversity of structures that can be manufactured. Here we demonstrate that a mixture of paramagnetic \\ and diamagnetic colloids in a ferrofluid can be used to create a variety of fractal aggregates in the presence \\ of a field. These aggregates exhibit growth both parallel and perpendicular to the field, a distinct departure \\ from the linear chains that are typical of dipolar assembly. The fractal dimension of these aggregates \\ displays a parabolic character as the ferrofluid concentration is increased and varies between $0.94 \pm$ \\ 0.03 and $1.54 \pm 0.03-a$ wider range than that which is seen when colloids are assembled using short- \\ range forces. This behavior is explained by examining how the ferrofluid concentration affects the \\ relative strength of the dipolar interactions between each type of particle. These dipolar fractal \\ aggregates may find use in the study of gelation via long-range forces or the preparation of gels that \\ can be activated using an external field.
}

Received 29th January 2013 Accepted 15th May 2013

DOI: $10.1039 / c 3 s m 50306 b$

www.rsc.org/softmatter leads primarily to chaining in the direction of the field. These chains do not yield network structures and increasing the particle fraction only results in crystalline phases..$^{20}$ Conversely, it is possible for a network-like phase to form in systems of colloids with permanent internal dipoles. In the absence of any external field, the predominantly chain-like aggregates formed by ferromagnetic particles can form loops and other branchpoints, which contribute to the formation of the gel phase. ${ }^{21}$

One of the main advantages of dipolar systems is the ability to tune the interaction strength outside the system (by modulating the external field strength). Therefore, it would be desirable to develop a system which gels in the presence of a field and reversibly disassembles when the field is removed. This system, more so than one composed of ferromagnetic particles, could find application in systems such as magnetorheological fluids-where switchable properties are desired. ${ }^{26,27}$ Here, we present a two-dimensional system of paramagnetic and diamagnetic colloids in a ferrofluid medium that forms fractal aggregates which grow in both the directions parallel and perpendicular to the external field. The particles in this system have a four-fold "valency" that is heterogeneous, meaning that the two sites along the field direction can only be occupied by like particles while the two sites perpendicular to the external field must be occupied by particles unlike the center particle, resulting in a square lattice packing. This should lead to a more complex phase diagram than a solely paramagnetic system. The branching seen in these structures will aid the formation of gels and provide a new way to study gelation via long-range interactions.

Previously, researchers have shown that aggregates with multipole symmetry ("flower" and "Saturn-ring" structures) as
Department of Chemical and Biomolecular Engineering, Rice University, 6100 Main St. MS 362, Houston, Texas 77005, USA. Tel: 713-348-6055; E-mail: biswal@rice.edu

$\dagger$ Electronic supplementary information (ESI) available. See DOI: 10.1039/c3sm50306b 
well as many crystalline phases form when a magnetic field is applied at various angles to the particle plane. ${ }^{28-30}$ Conversely, we explore how an in-plane magnetic field can be used to assemble fractal structures. We examine how altering the ferrofluid concentration affects the fractal dimension of the aggregates. We observe a parabolic trend in fractal dimension with increasing ferrofluid concentration. The fractal dimension increases from $1.20 \pm 0.02$ at low ferrofluid concentrations to $1.54 \pm 0.03$ at intermediate concentrations, then decreases to $1.09 \pm 0.02$ at higher concentrations. This behavior is explained by examining how the interparticle interactions vary across these conditions. By controlling which interaction is dominant, we are able to create a variety of branched structures. We also investigate the effect of tuning the overall bead concentration as well as the ratio of paramagnetic to diamagnetic particles in the system.

\section{Theory}

\subsection{Magnetic assembly in ferrofluid}

Colloids with intrinsic magnetic properties assemble in the presence of a magnetic field due to dipolar interactions. Additionally, microspheres without intrinsic magnetic properties (or with very small diamagnetic properties) can be assembled via magnetic field when suspended in a ferrofluid medium. In this phenomenon, the microspheres act as "magnetic holes" and acquire dipole moments antiparallel to the direction of the applied field. ${ }^{31,32}$ In effect, a system of diamagnetic particles in a magnetic medium behaves similarly to a system of paramagnetic particles in a non-magnetic medium.

Paramagnetic particles placed in a magnetic medium can exhibit dipoles either parallel or antiparallel to the direction of the external field, depending on the relative magnitudes of the medium and particle susceptibilities. The equation governing a particle's dipole in a magnetic medium is given by:

$$
\overrightarrow{\boldsymbol{m}}=4 \pi a^{3} K \overrightarrow{\boldsymbol{H}}_{\mathrm{ext}}
$$

where $a$ is the radius of the particle, $\boldsymbol{H}_{\text {ext }}$ is the applied magnetic field, and $K$ is the Clausius-Mossotti function relating the magnetic susceptibility of the particle to that of its surroundings

$$
K=\frac{\chi_{\mathrm{p}}-\chi_{\mathrm{f}}}{\chi_{\mathrm{p}}+2 \chi_{\mathrm{f}}+3}
$$

in this expression, $\chi_{\mathrm{p}}$ is the susceptibility of the particle and $\chi_{\mathrm{f}}$ is the susceptibility of the ferrofluid. The susceptibility of the paramagnetic particles is set during synthesis, while that of the diamagnetic particles is negligible and assumed to be zero. The ferrofluid susceptibility is easily tuned by adjusting the volume fraction of magnetic nanoparticles in the solution

$$
\chi_{\mathrm{f}}=\frac{\mu_{0} M_{\mathrm{s}}^{2} \phi_{\mathrm{f}} V}{3 k_{\mathrm{B}} T}
$$

where $\mu_{0}$ is the magnetic permeability of free space, $M_{\mathrm{S}}$ is the saturation magnetization of the bulk ferrofluid material, $\phi_{\mathrm{f}}$ is the volume fraction of magnetic nanoparticles in solution, and
$V$ is the volume of a single magnetic nanoparticle. It is simple to alter the dipole strengths of each particle type by changing the concentration of the ferrofluid. Increasing the ferrofluid concentration has the effect of increasing the diamagnetic dipole and decreasing the paramagnetic dipole.

To understand why imposing a magnetic field on a system of paramagnetic and diamagnetic particles in ferrofluid leads to two-dimensional aggregates, one can examine the expression describing the interaction energy between two particles, $\mathrm{i}$ and $\mathrm{j}$ :

$$
U_{\mathrm{i}, \mathrm{j}}(r, \alpha)=\frac{m_{\mathrm{i}} m_{\mathrm{j}}}{4 \pi \mu_{0} r^{3}}\left(1-3 \cos ^{2} \alpha\right)
$$

here, $r$ is the magnitude of the vector connecting the centers of the particles' dipoles and $\alpha$ is the angle between this vector and the direction of the magnetic field. Thus, for like particles the energetically favorable configuration is the formation of chains with their dipoles arranged head-to-tail $\left(\alpha=0^{\circ}\right)$. For all of our experimental conditions unlike particles will have dipoles of opposite signs. Therefore, the lowest energy configuration for unlike particle pairs is to stack at a $90^{\circ}$ angle relative to the direction of the applied field. A schematic of these relationships can be seen in Fig. 1.

The major factor that determines what types of structures form in these systems is the relative magnitude of the dimensionless dipole parameter, $\lambda$, for each particle pair. This parameter compares the relative magnitudes of the magnetic and thermal energies

$$
\lambda_{\mathrm{i}, \mathrm{j}}=\frac{-U_{\mathrm{i}, \mathrm{j}}(r, \alpha)}{k_{\mathrm{B}} T}
$$

Fig. 2 shows how tuning the ferrofluid susceptibility alters $\lambda$. In this plot, the ferrofluid susceptibility is normalized to the paramagnetic particle susceptibility $\left(X=\chi_{\mathrm{f}} / \chi_{\mathrm{p}}\right)$ and $\lambda$ is evaluated at the minimum interaction energy for each particle pair. Thus, the value of $r$ is taken to be the sum of both particles' radii. For like pairings, the angle is taken as $0^{\circ}$, while for the a)
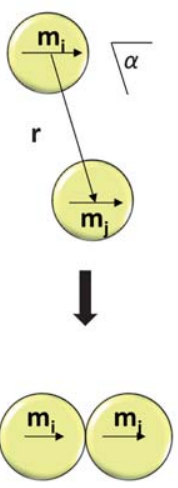

b)
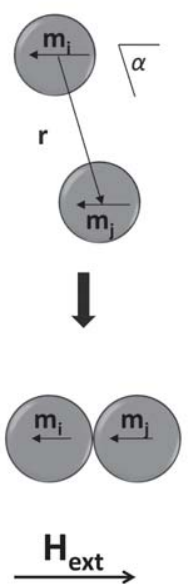

c)
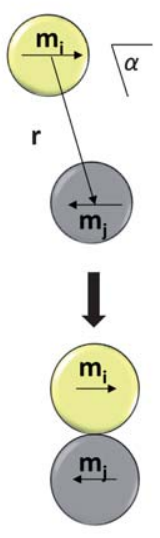

Fig. 1 Schematic of the three interaction types present in the experiments and the minimum energy configuration for each. Interactions between (a) two paramagnetic particles, (b) two diamagnetic particles, and (c) a paramagnetic and a diamagnetic particle in the presence of an external field, $\boldsymbol{H}_{\text {ext }}$. 


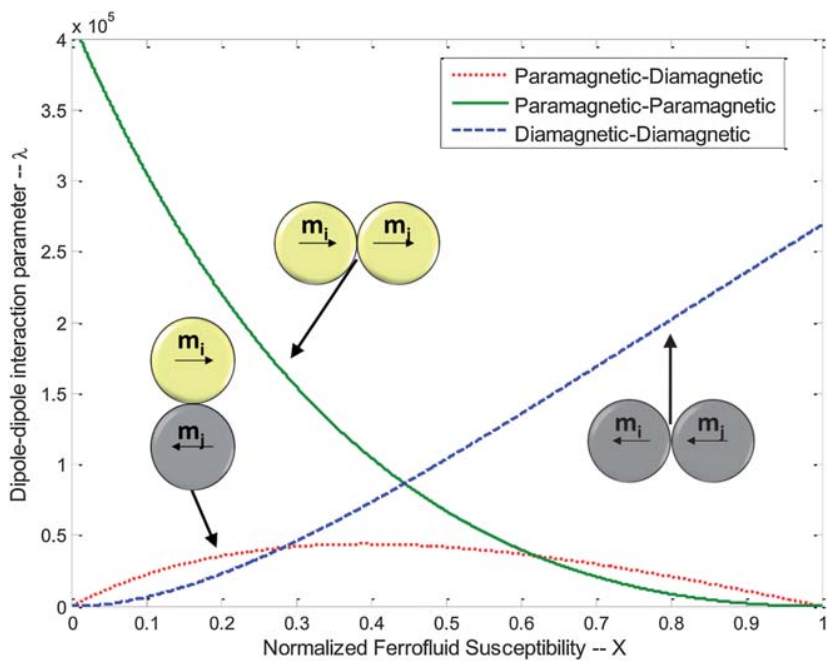

Fig. 2 The effect of changing the ferrofluid susceptibility on the strength of the dipolar interaction. The dimensionless dipole-dipole interaction, $\lambda$, is plotted against the ferrofluid susceptibility normalized by the paramagnetic particle susceptibility for the three types of interactions: paramagnetic-diamagnetic (dotted red line), paramagnetic-paramagnetic (solid green line), and diamagnetic-diamagnetic (dashed blue line).

case of an unlike particle pair the angle is assumed to be $90^{\circ}$. A field strength of 140 Gauss was used in the calculation, as this was the value used in our experiments. Altering the field strength will shift the curves up or down along the $y$ axischanging the magnitude of the interactions, but not their relative strengths.

This plot demonstrates the three main regions of the 2D assembly process. In the first $(0<X<0.3)$, the interaction between paramagnetic particles dominates and is the major determinant in aggregate formation. In the second region $(0.3<$ $X<0.7)$ the interaction between the three particle types are of similar magnitude and growth in both dimensions is expected to be of the same order. Finally, the third region $(X>0.7)$ is deemed to be that in which the dipolar interaction between the diamagnetic particles is dominant.

\subsection{Fractal dimension}

To describe the changes in morphology of these aggregates, we choose to analyze their fractal dimension. For colloidal aggregates, fractal dimension can be determined using the relationship between the number of beads in an aggregate and its radius of gyration ${ }^{33,34}$

$$
\left\langle R_{\mathrm{g}}(N)\right\rangle \sim N^{1 / D_{\mathrm{f}}}
$$

For a 2D system, the limiting cases are fractal dimensions of one and two. A chain-like aggregate would be expected to have a fractal dimension approaching one, while a sheet-like aggregate would have a fractal dimension closer to two. For example, consider the case of particles aggregating via short-range attractions. At one extremity is diffusion-limited cluster aggregation (DLCA), which occurs when the attraction between the particles is great enough that they bind irreversibly and diffusion is the limiting step. The fact that particles bind on contact results in relatively more open structures with an accepted 2D fractal dimension of $1.45 .^{35}$ On the other extreme is reaction-limited cluster aggregation (RLCA), where binding between particles is the limiting step. Particles are now able to diffuse further into the center of the cluster, creating denser aggregates with a 2D fractal dimension of $1.55 .^{35}$ Thus, the fractal dimension can provide a useful insight into the growth process that causes an aggregate with a certain morphology to form.

\section{Materials and methods}

\subsection{Experimental procedure}

Paramagnetic beads used for all experiments were DynabeadsM-270 Streptavidin (Invitrogen, Grand Island, NY) which were $2.8 \mu \mathrm{m}$ in diameter and coated with the protein streptavidin. These microspheres contain a polystyrene matrix interspersed with magnetic nanoparticles. As listed by the manufacturer, they have an iron content of $14 \%$, leading to a magnetic susceptibility of $6 \times 10^{-4} \mathrm{~m}^{3} \mathrm{~kg}^{-1}$. The diamagnetic particles are melamine resin beads (Sigma-Aldrich, St. Louis, MO) with a diameter of $3 \mu \mathrm{m}$. They are coated with a surface layer of carboxyl groups and are marked with a fluorescent FITC dye to allow them to be distinguished from the paramagnetic beads. Melamine resin was chosen as the material rather than the more common polystyrene because its density $(\rho=1.51 \mathrm{~g}$ $\mathrm{cm}^{-3}$ ) more closely matches that of the paramagnetic beads $\left(\rho=1.6 \mathrm{~g} \mathrm{~cm}^{-3}\right)$. Both settle quickly to the surface of the assembly cell under gravity, approximating a two-dimensional assembly process.

The ferrofluid used for these experiments was fluidMAG-PAS (Chemicell-GmbH, Berlin). Its saturation magnetization was confirmed by SQUID measurements to be $307 \mathrm{kA} \mathrm{m}^{-1}$, as shown in ESI. $\dagger$ These particles have a coating of (poly)acrylic acid with a hydrodynamic diameter of $50 \mathrm{~nm}$ as specified by the manufacturer. The magnetic core diameter was found by transmission electron microscopy to be between 10 and $20 \mathrm{~nm}$. The polymer brushes on the nanoparticles are terminated with carboxylate anions, which act to prevent the nanoparticles from aggregating even in the presence of an external magnetic field. This, along with the fact that the size of the nanoparticles is two orders of magnitude smaller than that of the microspheres, allows us to assume that the ferrofluid can be treated as a continuum with respect to the larger particles.

Experiments are conducted in a flow cell constructed using double-sided tape sandwiched between a glass slide and a coverslip. For each experiment, $40 \mu \mathrm{L}$ of solution are prepared. The ferrofluid particles are concentrated by first precipitating them from the aqueous stock solution using the antisolvent isopropyl alcohol (Sigma-Aldrich, St. Louis, MO) and centrifuged at $14000 \mathrm{rpm}$ for approximately one hour or until all nanoparticles had collected in the precipitate and the supernatant was clear. The nanoparticles are then redispersed to the desired concentration in $18.2 \mathrm{M} \Omega \mathrm{cm}$ deionized water (Millipore, Billerica, MA) with $0.5 \% \mathrm{v} / \mathrm{v}$ Tween-20 surfactant (SigmaAldrich, St. Louis, MO) to prevent nonspecific aggregation. To 
this solution are added the paramagnetic and diamagnetic microspheres in various ratios and overall bead concentrations to yield various surface coverage percentages on the glass surface.

Two ferrite bar magnets with dimensions 2 in. $\times 1$ in. $\times 0.5$ in. (McMaster Carr, Atlanta, GA) generate the magnetic field. A magnet spacing of 3 inches yielded a field strength of $140 \mathrm{G}$ in the flow cell. The system was observed using a digital camera (Hamamatsu, Hamamatsu City, Japan) attached to an inverted microscope (Olympus IX71, Olympus, Tokyo). Fluorescence images were captured using a mercury lamp (X-Cite 120, Lumen Dynamics, Ontario) combined with a FITC filter. Both fluorescent and bright field images were taken after the field had been applied for one hour. For each experiment, a series of 10-15 images were taken at $20 \times$ magnification that spanned the entire area of the flow cell. Each image contained anywhere from ten to thirty aggregates.

\subsection{Image processing}

The radius of gyration for each aggregate was determined via image processing done in MATLAB using the expression:

$$
R_{\mathrm{g}}{ }^{2} \equiv \frac{1}{N} \sum_{i=1}^{N}\left(\vec{R}_{i}-\vec{R}_{\mathrm{COM}}\right)^{2}
$$

where $\boldsymbol{R}_{i}$ is the position of each bead, $\boldsymbol{R}_{\mathrm{COM}}$ is the position of the aggregate center-of-mass (simply the average of all bead positions), and $N$ is the number of beads in the aggregate. The first step is to binarize the image such that all pixels that are part of the aggregate have a value of one, while all background pixels are zero. In order to separate which pixels belong to each bead, we use the MATLAB function 'watershed' as outlined by Gonzalez et al. ${ }^{36}$ Then, the locations of each bead's pixels are averaged to give its position and all bead positions of an aggregate are averaged to give its center of mass. Once all aggregates are analyzed, those comprised of the same number of beads are grouped and their radius of gyration averaged. Then, as in

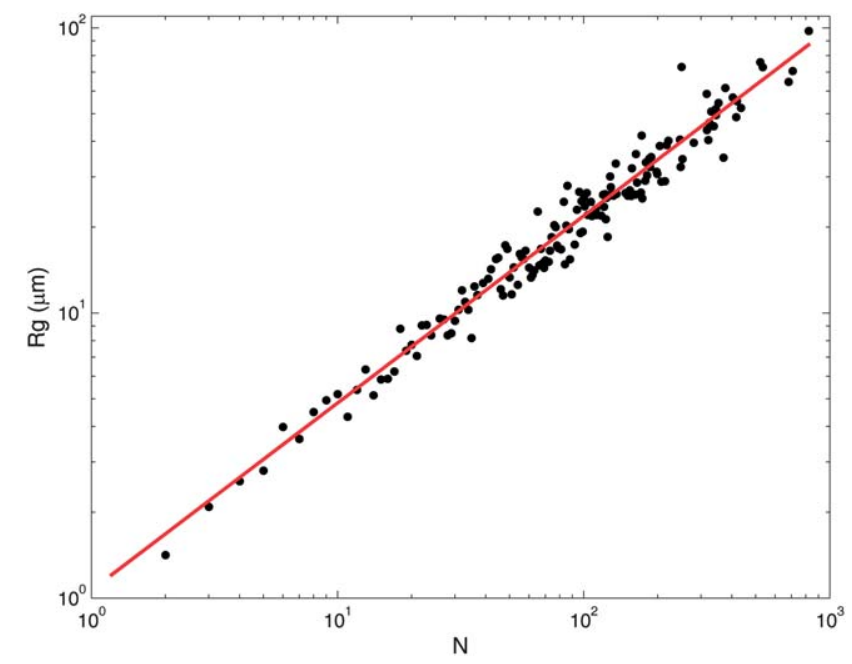

Fig. 3 Representative plot of the average radius of gyration vs. aggregate size for one experiment. The fractal dimension is given by the inverse slope of the linear regression on a log-log scale. eqn (6), the average radius of gyration can be plotted versus the aggregate size on a log-log scale and the fractal dimension determined from the inverse of the slope. Unlike mathematical fractals, natural fractals are only expected to be self-similar across a limited range of length scales. In this case, the radius of gyration vs. aggregate size data shows linear behavior only above a size of ten beads. As such, the fit was applied only to this subset of the data. A representative plot of $\left\langle R_{\mathrm{g}}\right\rangle v s$. $N$ can be seen in Fig. 3.

\section{Results}

\subsection{One-to-one ratio of diamagnetic and paramagnetic beads}

The results of the fractal dimension analysis for various ferrofluid concentrations, a $1: 1$ ratio of diamagnetic to paramagnetic beads, and an overall surface coverage of $14.6 \%$ can be seen in Fig. 4. The error bars in the figure represent the standard error of the slope obtained via linear regression of the radius of gyration data. The fractal dimension exhibits a parabolic behavior over the experimental range. The smallest ferrofluid concentration was chosen to be $30 \mathrm{mg} \mathrm{mL} \mathrm{mL}^{-1}$ (corresponding to a normalized susceptibility of $X=0.13$ ). Below this point the dipole moment of the diamagnetic particle is almost negligible and little aggregation is seen outside of the chaining of the paramagnetic particles. The fractal dimension at this concentration is $1.20 \pm 0.02$. The upper limit of $240 \mathrm{mg}$ $\mathrm{mL}^{-1}(X=1)$, is the point at which the ferrofluid susceptibility equals that of the paramagnetic particles and the dipole of the paramagnetic colloids is close to zero. For a value of $X=1$, aggregates formed with an average fractal dimension of $1.09 \pm$ 0.02. In between these two extremes, at $X=0.5$, the fractal dimension goes through a maximum value of $1.54 \pm 0.03$. The

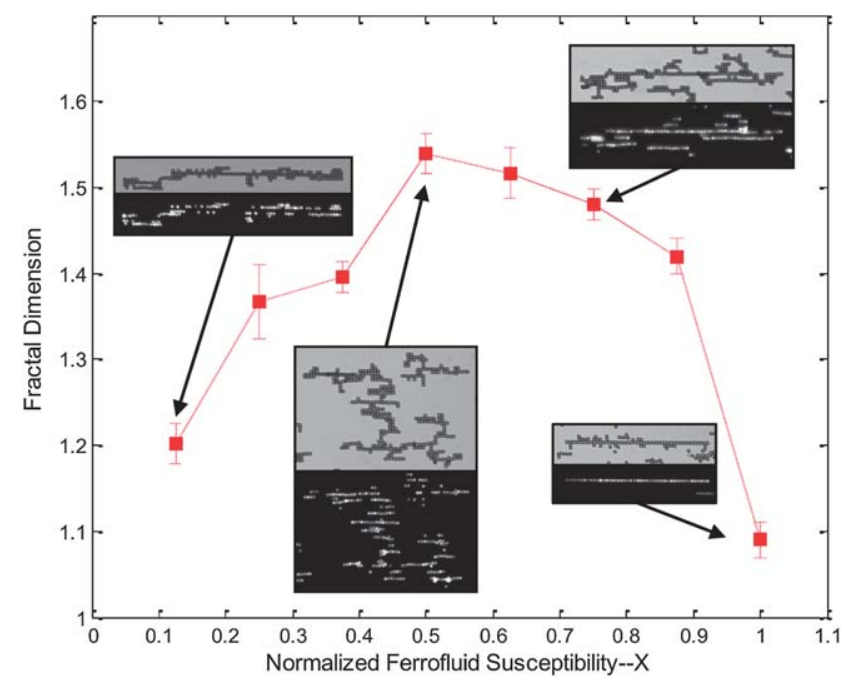

Fig. 4 Fractal dimension as a function of normalized ferrofluid susceptibility, with representative aggregates shown under both bright field and fluorescent illumination for certain values of $X$. The fractal dimension increases from a value of $1.20 \pm 0.02$ at low $X$ to a maximum of $1.54 \pm 0.03$, before decreasing again to $a$ minimum of $1.09 \pm 0.02$ at high $X$. All images taken at $20 \times$ magnification. Particle size is roughly $3 \mu \mathrm{m}$. 
composition of each type of particle in these aggregates was roughly $50 \%$ paramagnetic and $50 \%$ diamagnetic across all ferrofluid concentrations; thus, it is their arrangement as a fractal and not their composition which changes.

The images in Fig. 4 provide representative aggregates at select ferrofluid concentrations and can be used to explain the parabolic fractal dimension behavior. At low ferrofluid concentrations $(X<0.3)$, the paramagnetic-paramagnetic interaction is the strongest and the dipole of the diamagnetic particles is very weak. Thus, the mechanism for aggregation consists of paramagnetic chains, which form almost immediately upon the activation of the field. Only after this do the diamagnetic particles slowly start to assemble on either side of the chains. Indeed, we see that the positioning of the diamagnetic particles at the lowest ferrofluid concentration is often not in registry with the paramagnetic particles. The low strength of the dipolar interactions of a diamagnetic particle both with itself and with a paramagnetic particle means that the energetic penalty it must pay to align at angles other than $0^{\circ}$ or $90^{\circ}$ is not a limiting factor. This mechanism leads to highly linear aggregates with fractal dimensions closer to one.

As the ferrofluid concentration increases $(0.3<X<0.7)$, the interaction strength between paramagnetic particles decreases while simultaneously the interaction strength between the diamagnetic particles and between two unlike particles increases. The fact that the three interactions are of a similar order of magnitude in this region means that growth of chains in the direction of the field is no longer the principal aggregation mechanism. Instead, we note that small fractal-like aggregates form at the outset and these initial clusters then join together to create larger fractal assemblies. Additionally, the strength of the interaction between unlike particles goes through a maximum in this region and thus growth in the direction perpendicular to the field is greatest when $X$ is between 0.3 and 0.7. Subsequently, the fractal dimension increases to a maximum of $1.54 \pm 0.03$ at $X=0.5$ as these clusters have a more two-dimensional quality. Upon further increase of the ferrofluid concentration $(X>0.7)$ the aggregates appear once again to become linear. This is expected as the interaction between diamagnetic particles begins to dominate and the same principles apply as in the low ferrofluid concentration case. We observe that upon addition of the field, chains of diamagnetic particles form quickly and only after this do paramagnetic particles begin to assemble on these templates. Indeed, at the extreme value of $X=1$, the dipole moment of the paramagnetic particles is so low (essentially zero) that many of these particles do not incorporate into any aggregates.

At ferrofluid concentrations greater than $X=1$, the dipole of the paramagnetic particles will flip and become antiparallel to the field - parallel to the dipoles of the diamagnetic particles. After this point, the growth behavior will be fundamentally different: chains composed of both paramagnetic and diamagnetic particles should form, but no branching would occur. The particles at concentrations above $X=1$ lose their unique square valency. Instead, any growth in the direction perpendicular to the field would be close-packed 'bundling' as seen in systems of only one particle type at high bead concentrations.
To ensure that our measurements of fractal dimension were not time-dependent we tested samples allowed to aggregate under the field for four hours (data not shown). In these samples we did not observe any appreciable differences compared to the one hour measurements. Thus, after one hour, we believe our system has reached a kinetically trapped state with a stable fractal dimension.

\subsection{Effect of altering total surface coverage}

We also examined the effect of altering the overall bead concentration. Fractal dimension data for overall surface coverages ranging from $7.3 \%$ to $18.3 \%$ are shown in Fig. 5. As this data shows, the parabolic trend is not greatly influenced by the overall bead concentration, although the magnitude of the fractal dimension does differ between data sets. From this we conclude that the fractal dimension is a unique property related to the relative strengths of the three types of particle-particle interactions for a given ferrofluid concentration and system concentration is not a major influencing factor, at least within this moderate range.

\subsection{Effect of altering the ratio of diamagnetic to paramagnetic particles}

In order to better understand the driving force behind the aggregate assembly, we performed experiments where the ratio of diamagnetic to paramagnetic particles deviated from $1: 1$. Fig. 6 shows the results for ratios of $1: 1,1: 2$, and $1: 3$ diamagnetic to paramagnetic particles at the overall surface coverage of $14.6 \%$. The $1: 2$ curve shows a similar parabolic behavior as the $1: 1$ curve with two notable differences. First, the peak of the $1: 2$ curve occurs between $X=0.75$ and $X=0.88$ instead of $X=0.5$. Second, the magnitudes of the fractal dimensions of the $1: 2$ curve are generally lower than those of the $1: 1$ curve.

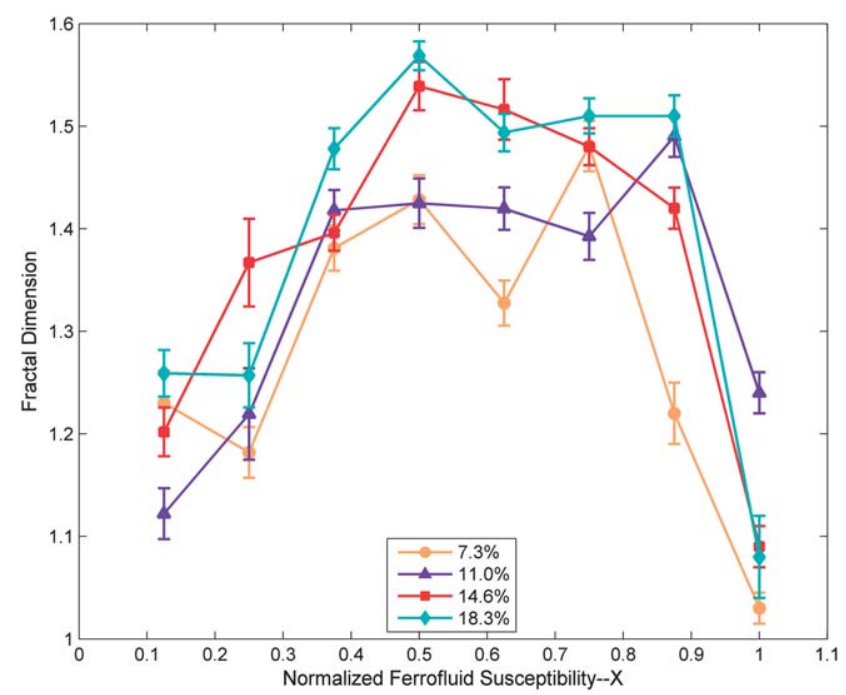

Fig. 5 Fractal dimension as a function of normalized ferrofluid susceptibility for 2D surface coverage's of $7.3 \%$ (orange circles), $11.0 \%$ (purple triangles), $14.6 \%$ (red squares), and $18.3 \%$ (teal diamonds). A general parabolic trend is seen for all cases, with the largest fractal dimensions occurring in the region $0.3<X<0.8$. 


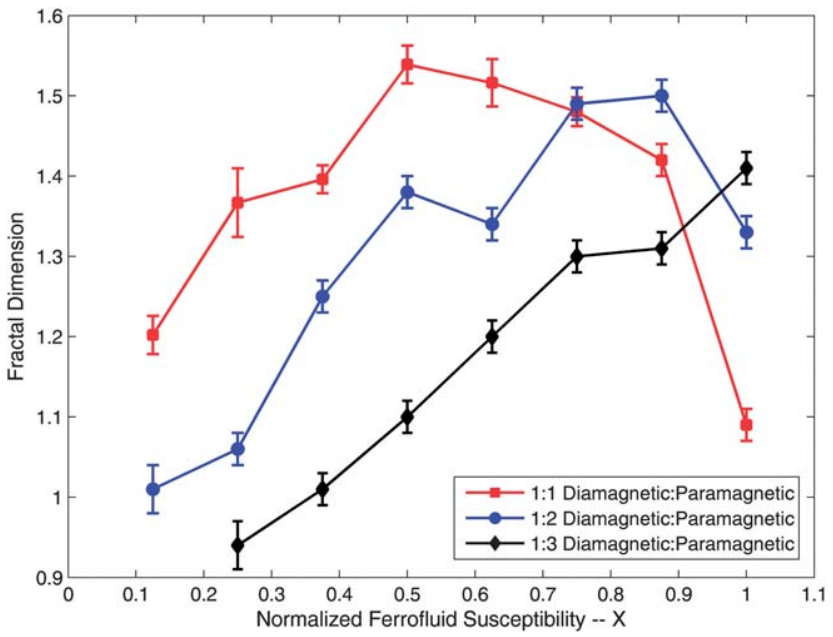

Fig. 6 Fractal dimension as a function of normalized ferrofluid susceptibility for different particle ratios: $1: 1$ (red squares), $1: 2$ (blue circles), and $1: 3$ (black diamonds) diamagnetic : paramagnetic. For all cases the overall surface coverage was $14.6 \%$. The parabolic behavior of the $1: 1$ ratio curve is seen in the $1: 2$ curve, albeit with a peak shifted to $X=0.88$ rather than 0.5 , but is not seen in the $1: 3$ curve.

If the interaction between unlike particles is the driving factor for the parabolic behavior it is reasonable to expect that the peak will shift when one particle is depleted. At a ratio of $1: 2$, it is more likely that a paramagnetic particle will encounter another paramagnetic particle than a diamagnetic particle-leading to more linear chains and aggregates with lower fractal dimensions. At high enough ferrofluid concentrations, the interaction strength of the paramagnetic particles has been reduced to the point where their relative concentration is not as important. The peak in fractal dimension occurs at this point. Furthermore, at a ratio of $1: 3$ diamagnetic to paramagnetic particles, there is no peak at all. There are simply not enough diamagnetic particles in the system to allow the diamagneticdiamagnetic interaction to become dominant and cause the decrease of fractal dimension at high ferrofluid concentrations. We also observe that at a ratio of $1: 3$ the fractal dimensions are even lower than either the $1: 1$ or $1: 2$ cases.

On the other hand, when the ratio is increased to $2: 1$ diamagnetic to paramagnetic, the behavior does not follow the parabolic trends of earlier experiments, as seen in Fig. 7. At low ferrofluid concentrations, the fractal dimensions are higher than expected and unpredictable. This is due to the relatively low interaction strength of the diamagnetic particles at these conditions. As mentioned previously, there is a lower energetic penalty to pay when the particles do not aggregate at either $0^{\circ}$ or $90^{\circ}$ in relation to other particles. Therefore, we see highly disorganized and close-packed structures rather than the square-lattice structures observed in other experiments. At higher ferrofluid concentrations (above $X=0.5$ ) there may be a recovery of the expected parabolic behavior, but it is not obvious.

\subsection{Comparison to fractal aggregates of systems using short- range attractions}

The utility of this system is readily apparent upon examination of the vast array of fractal structures exhibited over the range of

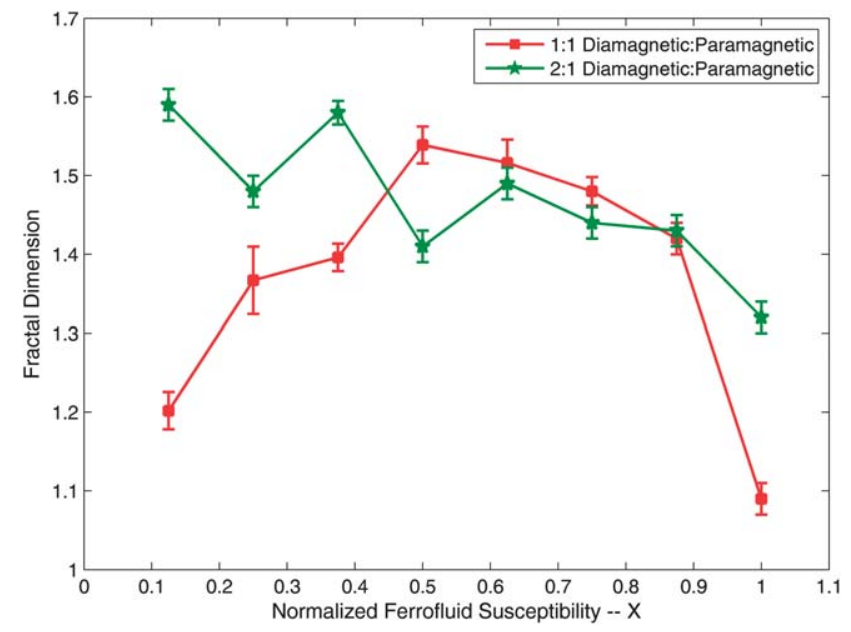

Fig. 7 Fractal dimension as a function of normalized ferrofluid susceptibility for the ratios $1: 1$ (red squares) and $2: 1$ (green stars) diamagnetic : paramagnetic. In both cases the surface coverage was $14.6 \%$. The parabolic nature of the fractal dimensions of the $1: 1$ curve is not evident in the $2: 1$ curve.

experimental conditions considered. We are able to achieve fractal dimensions from $0.94 \pm 0.03$ to $1.54 \pm 0.03$, a range much larger than that between the two extremes of aggregation via short-range forces noted previously. The difference between $\operatorname{DLCA}\left(D_{\mathrm{f}}=1.45\right)$ and RLCA $\left(D_{\mathrm{f}}=1.55\right)$ is roughly $20 \%$ of the range shown by the ferrofluid system with a mixture of paramagnetic and diamagnetic particles. Our system compares favorably with the range demonstrated by Helgesen, et al. ${ }^{4}$ using ferromagnetic colloids. They observed fractal dimensions from $1.16 \pm 0.05$ to $1.52 \pm 0.05$ in the limits of strong and weak dipolar interactions, respectively. However, unlike with clusters of ferromagnetic particles, our system has the added benefit of being tunable and reversible. These aggregates can disassemble simply by removing the external field.

\section{Conclusions}

We have reported a system of paramagnetic and diamagnetic particles assembled in a ferrofluid using an external magnetic field. The nature of the dipolar interactions between the two types of particles leads to the formation of fractal aggregates that grow in two dimensions. These structures are unique in that it has been difficult in the past to introduce branching into colloidal systems with dipole moments that depend on an external field. By simply changing the ferrofluid concentration, we are able to produce aggregates with fractal dimensions ranging from $1.09 \pm 02$ to $1.54 \pm 0.03$. Their fractal dimension changes predictably based on the relative strengths of the three different dipolar interactions. We can further extend the types of structures formed by changing the ratio of diamagnetic to paramagnetic particles. Using ratios of 1 : 2 and $1: 3$ diamagnetic to paramagnetic we achieve fractal dimensions down to $0.94 \pm 0.03$, however for a ratio of $1: 3$ parabolic fractal dimension behavior was no longer observed and was replaced by a linear trend. At low ferrofluid concentrations, a ratio of $2: 1$ diamagnetic to paramagnetic yielded aggregates that were 
largely disordered and did not demonstrate the four-fold valency seen in other experiments.

The ease with which we can tune the properties of this system offers a unique opportunity to study the formation of gels and networks via dipolar interactions. For example, one can envision investigating the percolation behavior of dipolar systems of varying fractal dimensions. Additionally, this system may be useful as a novel type of magnetorheological fluid. The branching seen in these aggregates may offer an advantage over fluids utilizing simple paramagnetic chaining by offering greater stabilization of the fluid against shear. Traditional magnetorheological fluids generally exhibit directionally dependent properties when subject to shear. This effect may be more easily controlled by our two-dimensional aggregates; however, we have yet to test the growth behavior in a bulk environment.

\section{Acknowledgements}

We are grateful to Kurt Kotzur for assistance with some of the data analysis. This work was supported by NSF grant CBET0955003.

\section{References}

1 J. S. R. Forrest and T. A. Witten, J. Phys. A: Math. Gen., 1979, 12, L109.

2 D. A. Weitz and M. Oliveria, Phys. Rev. Lett., 1984, 52, 1433.

3 D. A. Weitz, J. S. Huang, M. Y. Lin and J. Sung, Phys. Rev. Lett., 1985, 54, 1416.

4 G. Helgesen, A. T. Skjeltorp, P. M. Mors, R. Botet and R. Jullien, Phys. Rev. Lett., 1988, 61, 1736.

5 J. L. Carrillo, F. Donado and M. E. Mendoza, Phys. Rev. E: Stat., Nonlinear, Soft Matter Phys., 2003, 68, 061509.

6 G. A. Niklasson, A. Torebring, C. Larsson, C. G. Granqvist and T. Farestam, Phys. Rev. Lett., 1988, 60, 1735.

7 E. M. Furst, C. Suzuki, M. Fermigier and A. P. Gast, Langmuir, 1998, 14, 7334-7336.

8 S. L. Biswal and A. P. Gast, Phys. Rev. E: Stat., Nonlinear, Soft Matter Phys., 2003, 68, 021402.

9 D. Li, J. Rogers and S. L. Biswal, Langmuir, 2009, 25, 89448950.

10 D. Li, S. Banon and S. L. Biswal, Soft Matter, 2010, 6, 41974204.
11 C. c. Goubault, F. Leal-Calderon, J.-L. Viovy and J. r. m. Bibette, Langmuir, 2005, 21, 3725-3729.

12 E. Zaccarelli, J. Phys.: Condens. Matter, 2007, 19, 50.

13 P. J. Lu, E. Zaccarelli, F. Ciulla, A. B. Schofield, F. Sciortino and D. A. Weitz, Nature, 2008, 453, 499-503.

14 T. Ohtsuka, C. P. Royall and H. Tanaka, EPL, 2008, 84, 46002.

15 A. M. Puertas, M. Fuchs and M. E. Cates, Phys. Rev. Lett., 2002, 88, 098301.

16 C. M. Sorensen and A. Chakrabarti, Soft Matter, 2010, 7, 2284-2296.

17 K. Masschaele, J. Fransaer and J. Vermant, J. Rheol., 2009, 53, 1437-1460.

18 R. Blaak, M. A. Miller and J. P. Hansen, EPL, 2007, 78, 26002.

19 A. Goyal, C. K. Hall and O. D. Velev, Phys. Rev. E: Stat., Nonlinear, Soft Matter Phys., 2008, 77, 031401.

20 A.-P. Hynninen and M. Dijkstra, Phys. Rev. Lett., 2005, 94, 138303.

21 A. Goyal, C. K. Hall and O. D. Velev, Soft Matter, 2009, 6, 480484.

22 E. D. Gado, J. Phys.: Condens. Matter, 2010, 22, 5.

23 E. D. Gado and W. Kob, Soft Matter, 2010, 6, 1547-1558.

24 H. Schmidle, S. Jager, C. K. Hall, O. D. Velev and S. H. L. Klapp, Soft Matter, 2013, 9, 2518-2524.

25 S. Gangwal, A. Pawar, I. Kretzschmar and O. D. Velev, Soft Matter, 2010, 6, 1413-1418.

26 J. de Vicente, D. J. Klingenberg and R. Hidalgo-Alvarez, Soft Matter, 2011, 7, 3701-3710.

27 E. M. Furst and A. P. Gast, Phys. Rev. E: Stat. Phys., Plasmas, Fluids, Relat. Interdiscip. Top., 2000, 61, 6732-6739.

28 R. M. Erb, H. S. Son, B. Samanta, V. M. Rotello and B. B. Yellen, Nature, 2009, 457, 999-1002.

29 K. S. Khalil, A. Sagastegui, Y. Li, M. A. Tahir, J. E. S. Socolar, B. J. Wiley and B. B. Yellen, Nat. Commun., 2012, 3, 794.

30 A. Ray and T. M. Fischer, J. Phys. Chem. B, 2012, 116, 82338240.

31 A. T. Skjeltorp, Phys. Rev. Lett., 1983, 51, 2306.

32 A. T. Skjeltorp, J. Appl. Phys., 1985, 57, 3285-3290.

33 T. Vicsek, Fractal Growth Phenomena, World Scientific, Singapore, 1992.

34 P. Meakin, Fractals, scaling, and growth far from equilibrium, Cambridge University Press, 1998.

35 D. J. Robinson and J. C. Earnshaw, Phys. Rev. A, 1992, 46, 2045-2054.

36 R. C. Gonzalez, R. E. Woods and S. L. Eddins, Digital Image Processing Using MATLAB, Pearson Prentice hall, 2004. 